\title{
MODELO PARA EL CÁLCULO DE ÁREAS Y DE PERSONAL REQUERIDO EN LOS PROCESOS DE CENTRO DE DISTRIBUCIÓN DE UN OPERADOR LOGÍSTICO
}

Astrid Julieth Barreto Díaz', Mauricio Becerra Fernández²

Fecha de recibido: Julio 23 de 2015 | Fecha de aprobado: Septiembre 16 de 2015

\section{Resumen}

La estrecha relación existente entre la capacidad en las operaciones logísticas y la demanda a atender por parte del operador como empresa prestadora de estos servicios especializados, afectan el resultado esencialmente en términos de satisfacción al cliente y costos logísticos. En este artículo se plantea un modelo para el cálculo de áreas y de personal requerido en Centros de Distribución (CEDI), considerando el caso de un operador logístico representativo en el mercado colombiano, lo que brinda al operador logístico mencionado, elementos para planear con mayor eficiencia sus procesos, mediante la generación de planes de acción que faciliten el cumplimiento de la promesa de servicio al cliente.

Palabras claves: modelo de capacidad, centros de distribución, operador logístico.

1 Nació en Monterrey, Colombia. Es ingeniera Industrial de la Universidad Católica de Colombia. Integrante del Semillero Modelos Aplicados a la Logística y la Producción (ModeLoP), adscrito al Grupo de Investigación en Producción GIP de la Universidad Católica de Colombia. Actualmente se desempeña como Ingeniera de Logística y Mejoramiento en Suppla S.A. e-mail: ajbarreto05@ucatolica.edu.co

2 Nació en Bogotá, Colombia. Es Tecnólogo Industrial e Ingeniero de Producción de la Universidad Distrital Francisco José de Caldas. Obtuvo su título de Magister en Ingeniería Industrial en la Universidad Distrital Francisco José de Caldas, de Bogotá, Colombia. Actualmente estudia Doctorado en Modelado en Política y Gestión Pública en la Universidad Jorge Tadeo Lozano de Bogotá, Colombia, y PhD. in Model Based Public Planning, Policy Design and Management de la Universidad de Palermo, Italia. Se desempeña como profesor e investigador en las áreas de Logística, Producción, Modelado y Simulación en la Universidad Católica de Colombia, Director del Grupo de Investigación en Producción GIP. e-mail: mbecerra@ucatolica.edu.co, mauriciobecerrafernandez@gmail.com 


\title{
MODEL FOR THE CALCULATION OF PERSONNEL REQUIRED AREAS AND DISTRIBUTION PROCESSES OF A LOGISTICS CENTER
}

\begin{abstract}
The tight relationship that exists between the capacity owned in the logistics operations and the demand to be attended -by the operator as a specialized provider industry of this kind of services- affects the desired result; essentially in terms of customer's satisfaction and logistics costs. In this article a model for the calculation of areas and required personal in Distribution Centers is suggested, considering the case of a representative logistics operator in the Colombian market, which offers elements for the mentioned logistics operator to plan with more efficiency its own processes, via the generation of action plans that facilitates the promise's fulfillment of customer service.
\end{abstract}

Keywords: capacity model, distribution centers, logistics operator.

\section{MODELO PARA O CÁLCULO DE ÁREAS E DE PESSOAL, REQUERIDO NOS PROCESSOS DE UM CENTRO DE DISTRIBUIÇÃO E OPERADOR LOGÍSTICO}

\begin{abstract}
Resumo
A estreita relação existente entre a capacidade das operações logísticas e a demanda a atender por parte do operador como empresa prestadora destes serviços especializados, afetam o resultado essencialmente em termos de satisfação do cliente e custos logísticos. Neste artigo propõe-se um modelo para o cálculo de áreas, e de pessoal requerido em Centros de Distribuição (CEDI), considerando o caso de um operador logístico representativo no mercado colombiano, o que brinda ao operador logístico mencionado, elementos para planejar com maior eficiência seus processos, mediante a geração de planos de ação que facilitem o cumprimento da promessa de serviço ao cliente.
\end{abstract}

Palavras-chave: modelo de capacidade, centros de distribuição, operador logístico. 


\section{INTRODUCCIÓN}

El cálculo de capacidades es un punto clave en los procesos de planeación de cualquier organización con el objetivo principal de conocer cómo se atenderá la demanda de sus productos. Los procesos logísticos no son la excepción, es por esto que la eficiencia en la cadena de suministros tiene relación directa con el cálculo de capacidades para determinar los recursos que son necesarios para los procesos en diversas operaciones con diferentes configuraciones y tipos de productos.

Un operador logístico surge como una empresa que gestiona las diversas actividades en la cadena de suministro, por medio de servicios especializados prestados a diferentes mercados por medio del transporte nacional e internacional, agenciamiento aduanero, almacenamiento de despacho y distribución de mercancías, entre otros (Suppla S.A., 2014). Dada la complejidad que puede acarrear el manejo de grandes variedades de productos y con diversas características operativas, un operador logístico requiere realizar el cálculo de capacidades para gestionar los recursos a asignar con miras a cumplir con la promesa de servicio de cara al cliente y al menor costo posible.

De no realizar correctamente este cálculo de capacidades en los centros de distribución de mercancías administrados por el operador logístico, se pueden presentar esencialmente pérdidas de tiempo, espacio, existencias y control sobre las operaciones y los costos involucrados. Para Mora (2011):

Las instalaciones, equipo y técnicas para el almacenamiento cambian mucho según la naturaleza de los materiales que se van a manejar, todas aquellas características del material como lo son tamaño, peso, durabilidad, duración en estantería y tamaño de los lotes son factores importantes que se deben tomar en cuenta para cualquier diseño de un sistema de almacenamiento.

Considerando que un operador logístico puede llegar a gestionar varias cuentas o clientes, se deben involucrar las variables descritas para el diseño tanto de sus procesos como de las instalaciones que soportan su ejecución. Mediante este artículo se toman modelos de referencia para el cálculo de capacidades, de tal manera se logre proponer un modelo matemático para el cálculo de capacidades en centros de distribución, tomando como caso de estudio un operador logístico representativo en el mercado colombiano.

\section{MODELOS PARA EL CÁLCULO DE CAPACIDADES}

\section{Definición de capacidad}

Dentro de las definiciones sobre el concepto de capacidad se resaltan las siguientes:

Kalenatic y Blanco (1993) argumentan que la capacidad técnica:

Está determinada por la potencialidad (máximo rendimiento posible que pueden obtener estas en su desempeño; o sea, la máxima productividad alcanzable en el $100 \%$ del tiempo disponible para ello), que posee un sistema, unidad estructural o elemento, en realizar una determinada producción $\mathrm{y} / \mathrm{o}$ un servicio en un periodo de tiempo dado.

Para Sipper y Bulfin, la capacidad se define como "cuánto puede fabricar un sistema de producción. La definición de capacidad depende del sistema ya sea de manufactura y/o de servicios" (1997). Según Krajewski et ál. (2008) "la capacidad es la tasa de producción máxima de una instalación. Esta última puede ser una estación de trabajo o una organización entera".

Para Hopp y Spearman (2008):

la capacidad es el límite máximo en el rendimiento de un proceso de producción. En la mayoría de los casos, el trabajo de liberación en el sistema o por encima de la capacidad hace que el sistema se vuelva inestable, es decir, se acumule sin límite el Work in Process (WIP) o trabajo en proceso. Sólo los sistemas muy especiales pueden operar de manera estable a su máxima capacidad.

Chase et ál. (2009) definen la capacidad desde la perspectiva de los negocios como:

la cantidad de producción que un sistema puede conseguir durante un periodo específico. Es un establecimiento de servicios, esto puede representar la cantidad de consumidores que puede atender entre el medio día y la una de la tarde. En la producción, la capacidad podría ser el número de automóviles que puede producirse durante un turno. 
Para Collier y Evans(2009), la capacidad es:

la suficiencia de un recurso de manufactura o de servicio, como una instalación, un proceso, una estación de trabajo o una pieza de equipo, para lograr su propósito durante un periodo determinado. La capacidad se puede considerar en una de dos formas: (1) como el índice máximo de producción por unidad de tiempo, o (2) como unidades de la disponibilidad del recurso.

En el cálculo de las capacidades son de considerar los tipos de períodos de planeación a los cuales son aplicables, que para Chase et. al (2009) son: i) largo plazo considerando tiempo (más de un año) para obtener o entregar los recursos que se utilizan para la producción y donde es importante la participación de la alta gerencia, ii) mediano plazo considerando periodos mensuales o trimestrales teniendo en cuenta que diferentes alternativas (subcontratación, recorte personal, nuevas herramientas) pueden alterar la capacidad.

En el análisis de las capacidades se deben considerar las diferencias y similitudes existentes entre la prestación de servicios (como es el caso de los servicios logísticos) y la fabricación de productos, como se muestra en la tabla 1:

Tabla 1. Características de los procesos de servicios y de manufactura

\begin{tabular}{|l|l|l|}
\hline \multicolumn{1}{|c|}{ ASPECTO } & \multicolumn{1}{|c|}{ SERVICIO } & \multicolumn{1}{c|}{ MANUFACTURA } \\
\hline Tangibilidad & Intangibles: los servicios se viven. & Tangibles: los bienes se consumen. \\
\hline Tiempo & $\begin{array}{l}\text { No pueden ser almacenados para ser } \\
\text { utilizados posteriormente. }\end{array}$ & $\begin{array}{l}\text { Se pueden almacenar en bodegas dependiendo } \\
\text { de su caducidad. }\end{array}$ \\
\hline Capacidad & $\begin{array}{l}\text { Debe haber capacidad para cuando se } \\
\text { necesite. }\end{array}$ & $\begin{array}{l}\text { El cliente puede obtener bienes almacenados } \\
\text { producto de la anticipación a la demanda. }\end{array}$ \\
\hline Ubicación & Debe ubicarse cerca al cliente. & $\begin{array}{l}\text { Primero se lleva a cabo la producción y } \\
\text { posteriormente se distribuyen los bienes al } \\
\text { consumidor. }\end{array}$ \\
\hline Volatilidad de \\
la demanda & $\begin{array}{l}\text { Más alta debido a: } \\
\text { Clientes interactúan directamente con } \\
\text { el sistema de producción. (diferentes } \\
\text { necesidades y nivel de experiencia con el } \\
\text { sistema). } \\
\text { Demanda directamente afectada por el } \\
\text { comportamiento del consumidor. }\end{array}$ & $\begin{array}{l}\text { Menor debido a: } \\
\text { Se puede emplear el inventario para atender la } \\
\text { demanda. } \\
\text { Los clientes tienen mayor grado de interacción } \\
\text { con el sistema de producción ya que al inicio se } \\
\text { establecen las especificaciones del producto. } \\
\text { Posibilidad de realizar estimaciones sobre el } \\
\text { comportamiento de la demanda, facilitando los } \\
\text { procesos de planeación. }\end{array}$ \\
\hline $\begin{array}{l}\text { La naturaleza intangible del servicio hace } \\
\text { más difícil impedir que estos copien un } \\
\text { concepto de negocios, distribución de planta } \\
\text { y diseño de un encuentro de servicio. }\end{array}$ & $\begin{array}{l}\text { Protegidos bajo leyes de derechos de autor de } \\
\text { acuerdo a las especificaciones o características } \\
\text { propias de diseño. }\end{array}$ \\
\hline
\end{tabular}

Fuente: Chase, Jacobs, \& Aquilano, 2009, p. 444 y Collier \& Evans, 2009, p. 11

La cadena de suministro se orienta hacia todas las partes involucradas en los procesos para la satisfacción de una necesidad específica y se enfoca en estos procesos que se desarrollan antes, durante y después de entregar un producto $y / 0$ servicio al cliente. Para que esto sea efectivo, se busca mantener balanceada la capacidad de los diversos actores, en los ciclos de demanda, abasto, fabricación y reabastecimiento (Chopra \& Meindl, 2008).
Dentro de la cadena de suministro la capacidad es medida teniendo en cuenta que integra todas las actividades y procesos logísticos para transformar los materiales y servicios en bienes y/o productos entregados al cliente. Para mantener un alto promedio de utilización, se invierte en capacidad adicional y procesos flexibles. En cuanto al inventario en los almacenes, se busca minimizar su nivel en toda la cadena para reducir el costo con el efecto de que la capacidad requerida en estos almacenes sea menor (Heizer \& Render, 2009). 
Dentro de la planeación logística se abordan cuatro áreas principales de problemas relacionadas con el dimensionamiento de la capacidad, tales como los identifica Ballou (2004): "niveles de servicio al cliente, ubicación de instalaciones, decisiones de inventario y decisiones de transporte".

Con base en estas definiciones y aspectos a considerar, la capacidad en centros de distribución se asocia a las habilidades que se tienen en una instalación para llevar a cabo las operaciones logísticas como un servicio a su interior desde el recibo, pasando por el almacenamiento y finalmente en el despacho, para garantizar el cumplimento de los requerimientos del cliente y los organismos de control, mediante la asignación adecuada de recursos y sin descuidar el costo asociado a su desarrollo.

\section{Tipos de capacidad}

Para los medios de trabajos expresados como máquinas individuales $o$ en grupos e incluso instalaciones, la capacidad se puede clasificar por su magnitud como se muestra en la figura 1:

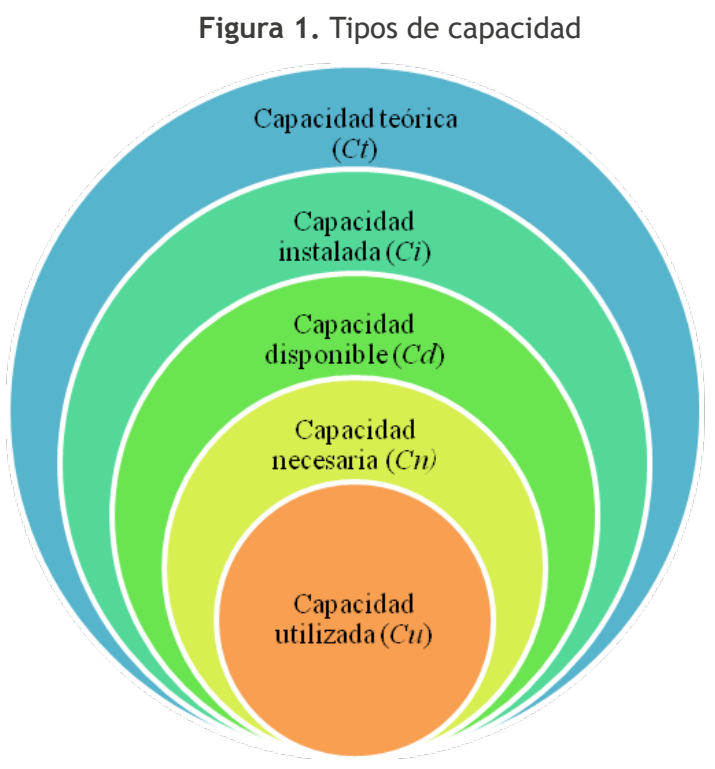

Fuente: Kalenatic y Blanco, 1993.

La capacidad tiene diferentes maneras de expresarse (Heizer y Render, 2009):

- Unidades de tiempo (horas por máquina, horas por unidad, etc.)
- Unidades energéticas (caballos de fuerza, kilovatios, etc.)

- Unidades Monetarias (pesos, dólares, marcos, etc.)

\section{Modelo de capacidades en manufactura}

Como principal referente se considera el modelo planteado por Kalenatic y Blanco (1993), en el cual se calculan cada uno de los tipos de capacidades como se muestra a continuación.

\section{Capacidad teórica}

Es aquella que potencia y alcanza los máximos resultados de producción. Representa la producción posible, si todas las máquinas y equipos estuvieran al $100 \%$ y en funcionamiento ininterrumpido. Puede ser sostenida para muy cortos periodos de tiempo, así como pocas horas al día o pocos días al mes.

$$
\mathrm{c}_{\mathrm{t}}=\sum_{\mathrm{i}=1}^{\mathrm{m}} \mathrm{n}_{\mathrm{i}} * 365 * 24\left(\frac{\text { horas }}{\text { año }}\right)
$$

Donde:

- $\mathrm{i}$ = número de sitios de trabajo $(\mathrm{i}=1,2, \ldots \mathrm{m})$

- $\mathrm{n}_{\mathrm{i}}=$ número de sitios de trabajo del mismo tipo

- 365 [días en el año (día/año)]

- 24 [horas en el día (horas/día)]

\section{Capacidad Instalada}

Representa la producción posible si todas las máquinas y equipos estuvieran al $100 \%$ y en funcionamiento ininterrumpido menos las pérdidas estándar por mantenimiento:

$$
\begin{gathered}
\mathrm{c}_{\mathrm{i}}=\sum_{\mathrm{i}=1}^{\mathrm{m}} \mathrm{n}_{\mathrm{i}} * 365 * 24-\sum_{\mathrm{i}=1}^{\mathrm{m}} \mathrm{n}_{\mathrm{i}} * \mathrm{~g}_{\mathrm{i}}\left(\frac{\text { horas }}{\text { año }}\right) \\
\mathrm{G}_{1}=\sum_{\mathrm{i}=1}^{\mathrm{m}} \mathrm{n}_{\mathrm{i}} * \mathrm{~g}_{\mathrm{i}}\left(\frac{\text { horas }}{\text { año }}\right)
\end{gathered}
$$

Donde:

- $\mathrm{g}_{\mathrm{i}}=$ pérdidas estándar por mantenimiento preventivo (horas/sitio de trabajo)

- $\mathrm{G}_{1}=$ pérdidas estándar por mantenimiento preventivo de todos los puestos de trabajo 


\section{Capacidad disponible}

Es menor que la capacidad instalada y depende de las condiciones de producción, administración y organización en que esta se desempeña. En comparación con la capacidad instalada, la capacidad disponible se ve disminuida en relación con los días hábiles del año, el número de turnos y su duración, las horas perdidas por ausentismo de los trabajadores, las pérdidas originadas por factores organizacionales y por otros factores influyentes externos:

$\mathrm{C}_{\mathrm{d}}=\sum_{\mathrm{i}=1}^{\mathrm{m}} \mathrm{n}_{\mathrm{i}} * \mathrm{~d}_{\mathrm{h}} * \mathrm{~h}_{\mathrm{t}} * \mathrm{n}_{\mathrm{t}}-\left(\mathrm{G}_{1}+\mathrm{G}_{2}+\mathrm{G}_{3}+\mathrm{G}_{4}\right) \quad\left(\frac{\text { horas }}{\text { año }}\right)$

Donde:

- $\mathrm{d}_{\mathrm{h}}=$ días hábiles en el año (día/año)

- $h_{t}=$ número de horas por turno

- $\mathrm{n}_{\mathrm{t}}=$ número de turnos según las condiciones de producción

- $\mathrm{G}_{2}=$ pérdidas estándar por no asistencia de los trabajadores (horas/año)

- $\mathrm{G}_{3}=$ pérdidas estándar de tiempo por factores organizacionales (horas/año)

- $\mathrm{G}_{4}=$ pérdidas estándar en el tiempo por factores externos naturales (horas/año)

\section{Capacidad necesaria}

Consiste en la capacidad para aprovechar las posibilidades y las exigencias del mercado. Indica cuanto se debe utilizar para un determinado plan de producción y puede ser mayor, igual o menor que la capacidad instalada o disponible:

$$
\mathrm{C}_{\mathrm{n}}=\sum_{\mathrm{i}=1}^{\mathrm{m}} \sum_{\mathrm{j}=1}^{\mathrm{p}}\left(\mathrm{Qp}_{\mathrm{ij}} * \mathrm{nr}_{\mathrm{ij}} \pm \mathrm{Est}_{\mathrm{ij}}\left(\frac{\text { horas }}{\text { año }}\right)\right.
$$

Donde:

- $j$ = variedad de productos $(j=1,2, \ldots, p)$

- $\mathrm{Qp}_{\mathrm{ij}}=$ cantidad planeada de productos tipo $\mathrm{j}$ en el puesto de trabajo i (unidades/año)

- $\mathrm{nr}_{\mathrm{ij}}=$ norma técnica del producto $\mathrm{j}$ en el sitio de trabajo tipo i (horas/unidad)

- $\mathrm{Est}_{\mathrm{ij}}=$ ejecución estándar de la norma por encima o por debajo (horas/unidad)

\section{Capacidad utilizada}

Refleja su real utilización para un determinado periodo de tiempo; es decir, la producción realizada expresada en las mismas unidades de medida en que se han calculado, la capacidad instalada, disponible y necesaria a los efectos de comparación y correspondencia con los factores perturbadores:

$$
c_{\mathrm{u}}=\sum_{\mathrm{i}=1}^{\mathrm{m}} \sum_{\mathrm{j}=1}^{\mathrm{p}}\left(\mathrm{Qr}_{\mathrm{ij}} * \operatorname{tr}_{\mathrm{ij}}\right)\left(\frac{\text { horas }}{\mathrm{año}}\right)
$$

Donde:

- $\mathrm{Qr}_{\mathrm{ij}}=$ cantidad del producto $\mathrm{j}$ elaborada en el sitio de trabajo tipo i (unidades/año)

- $\operatorname{tr}_{i j}=$ horas de trabajo realmente utilizadas por unidad de producto (horas/unidades)

\section{MATERIALES Y MÉTODOS}

Para el desarrollo del modelo se inició con la caracterización de las operaciones logísticas, posteriormente se realizó el levantamiento de la información de las operaciones identificadas mediante entrevistas y toma de tiempos, se continuó con la formulación matemática para el cálculo de capacidades y la aplicación del modelo en el operador logístico, finalmente, se analizaron los resultados obtenidos (ver figura 2).

Figura 2. Etapas para el desarrollo del modelo de capacidades

$\begin{gathered}\text { Identificación de las características de las } \\ \text { operaciones logísticas }\end{gathered}$
$\begin{array}{r}\text { Levantamiento de la información de las operaciones } \\ \text { en centro de distribución }\end{array}$
\[ \]
Formulación del modelo para el cálculo de
capacidades
$\downarrow$
Aplicación del modelo al caso de estudio del
operador logístico
$\downarrow$
Análisis de resultados del modelo

Fuente: elaboración propia

Características de las operaciones logísticas y levantamiento de información

En las características de las operaciones logísticas se identificaron los elementos que se muestran en la figura 3. 
Figura 3. Esquema para identificación de las características de las operaciones

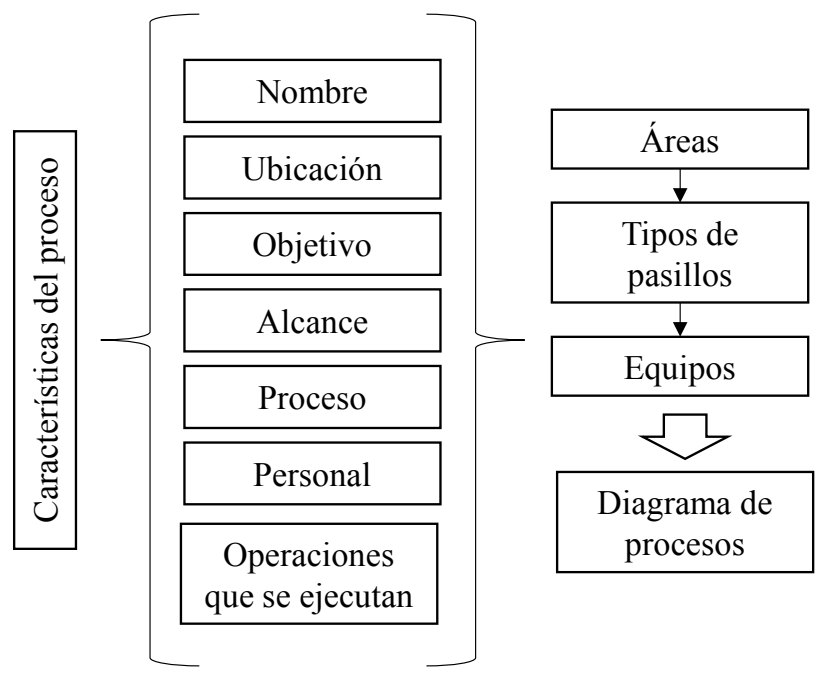

Fuente: elaboración propia.

En las operaciones logísticas estudiadas se identificaron tres principales procesos:

- Recibo de productos.

- Almacenamiento.

- Alistamiento y despacho.

Los diagramas de proceso de estas actividades, se muestran en las figuras 4, 5 y 6 .

Figura 4. Diagrama de proceso de recibo de productos

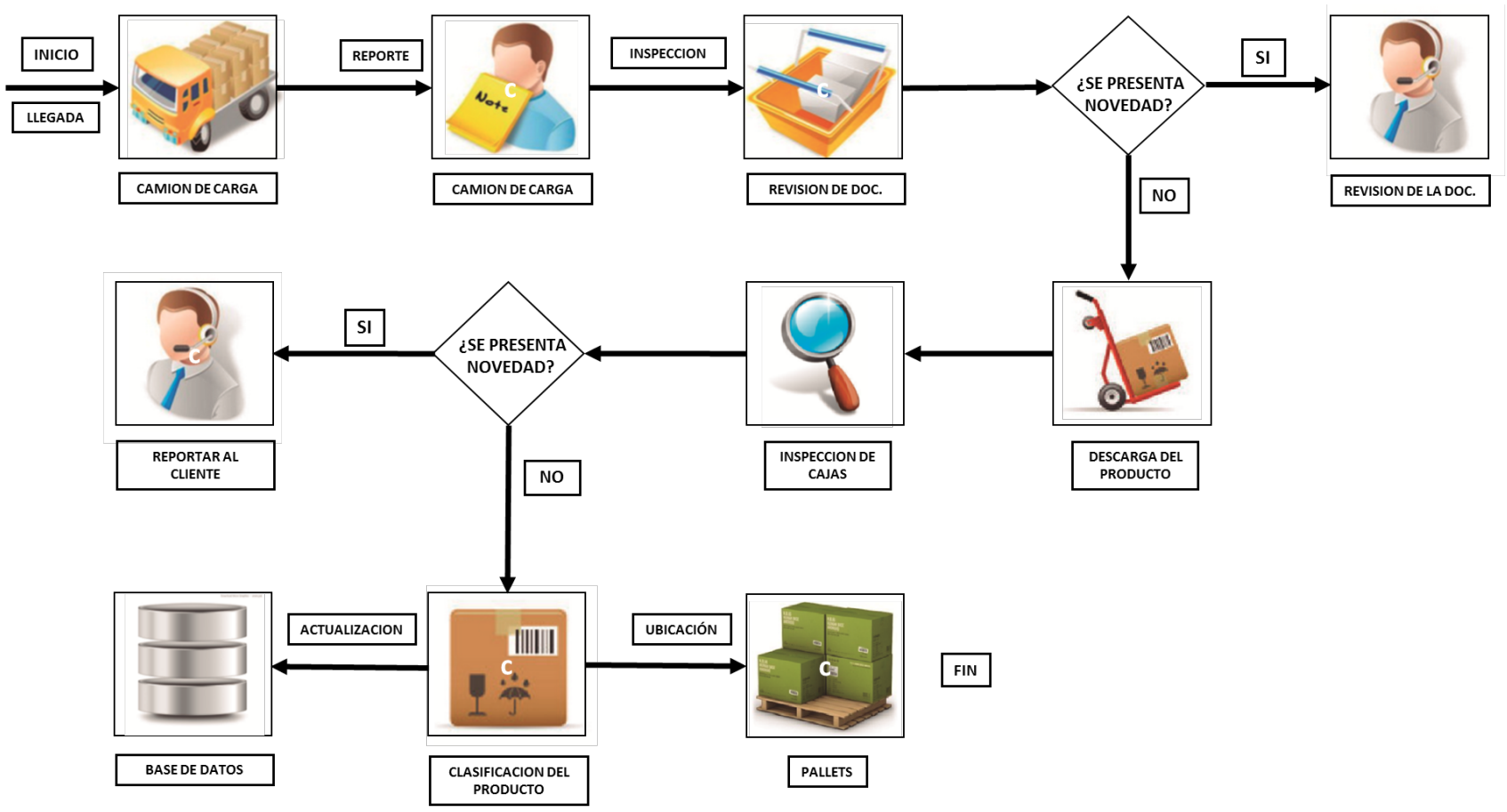

Fuente: elaboración propia 
Figura 5. Diagrama de proceso de almacenamiento

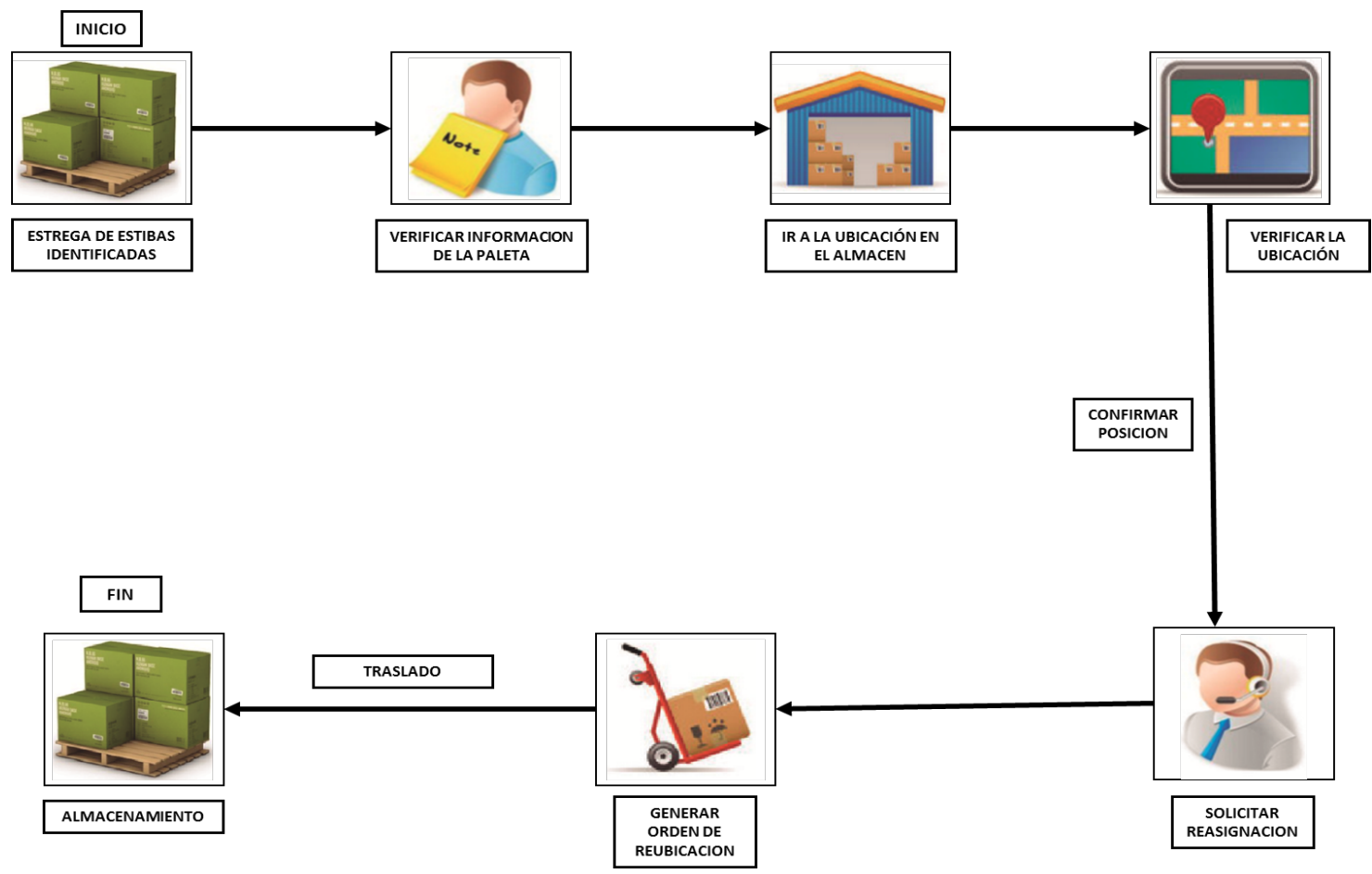

Fuente: elaboración propia.

Figura 6. Diagrama de proceso de alistamiento y despacho

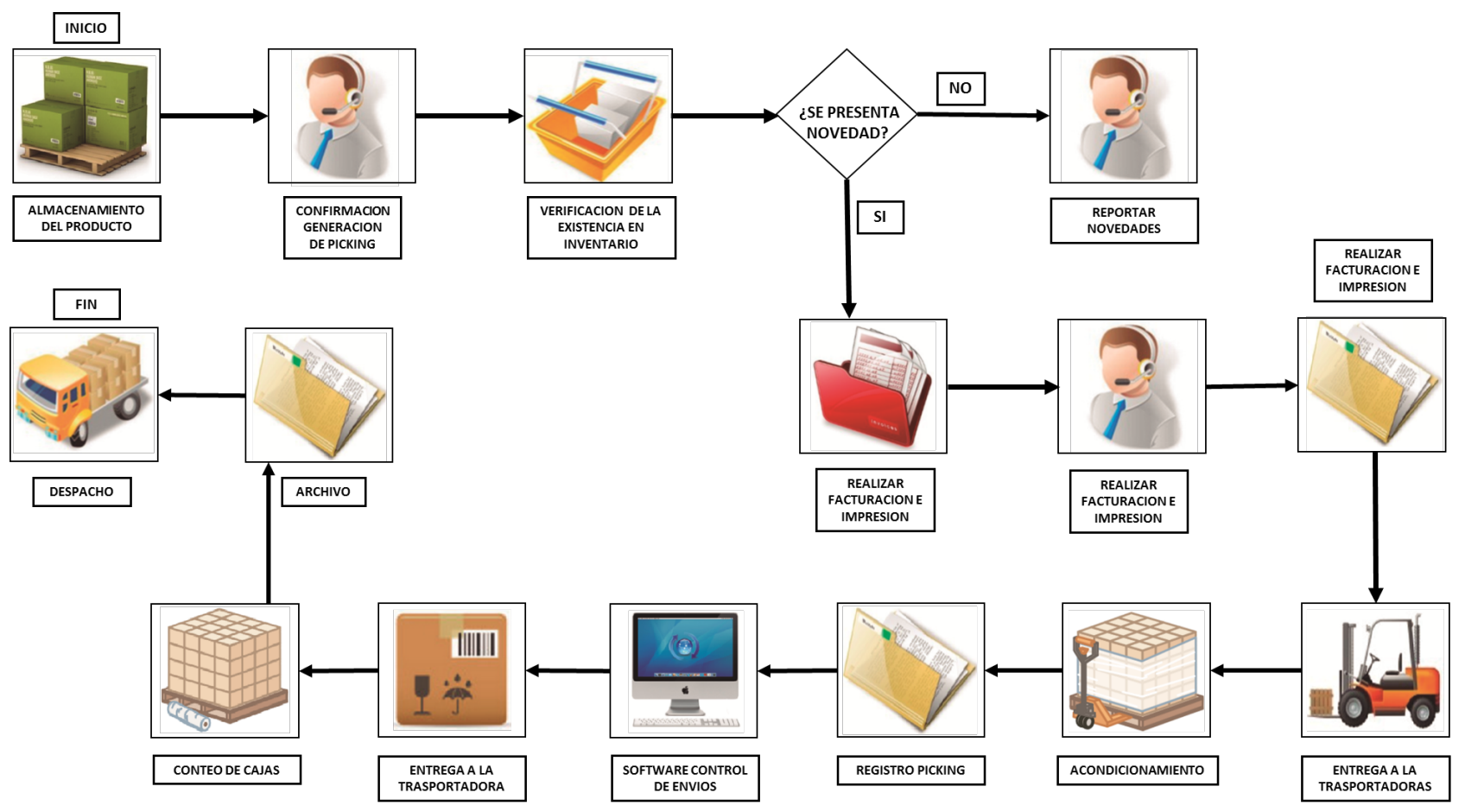


Una vez identificados los procesos se realizaron entrevistas con el personal involucrado, además, se revisaron registros de tiempos históricos y el levantamiento de nuevos registros con el objetivo de garantizar que se contaba con la información para el desarrollo del cálculo de capacidades en cuanto a áreas y personal.

\section{Cálculo de áreas}

El cálculo de áreas se fundamenta en las que se muestran en la figura 7.

Figura 7. Cálculos realizados con respecto a las áreas

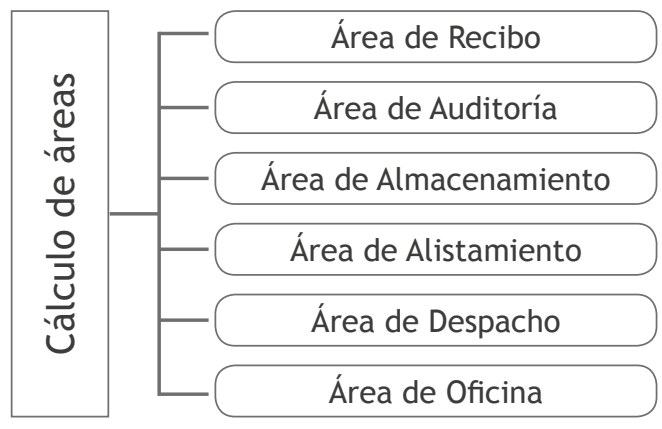

Fuente: elaboración propia.

Área de Recibo

Área del Pallet (APT)

$A P T=L E * A E\left\lfloor m^{2} /\right.$ pallets $\rfloor$

Donde:

- $L E=$ largo del pallet $[\mathrm{m}]$

- $A E=$ ancho del pallet $[\mathrm{m}]$

Área de Circulación del Pasillo $(A C P)$

$A C P=L P * A P\left[\mathrm{~m}^{2}\right]$

Donde:

- $L P=$ largo del pasillo $[\mathrm{m}]$

- $A P=$ ancho del pasillo $[m]$

Área de Recibo $(A R)$

$A R=(N P R * A P T)+[(N P R * P A R) * A P T]+A C P\left[m^{2}\right]$

Donde:

- $N P R=$ número de pallets recibidos [pallets]

- $P A R=$ porcentaje de acumulación en recibo [\%]
Área de auditoría

Área de Auditoría $(A A)$

$A A=(N P A * A P T) *[(N P A * P A A) * A P T]+A C P\left[m^{2}\right]$

Donde:

- $N P A=$ número de pallets auditadas [pallets]

- $P A A=$ porcentaje de acumulación en auditoría [\%]

\section{Área de Almacenamiento}

Para el cálculo del área de almacenamiento es necesario definir qué es un cuerpo de almacenamiento, con varios cuerpos de almacenamiento conformados, se logra constituir una línea o bahía de almacenamiento como se muestra en la figura8.

Figura 8. Línea de almacenamiento constituida por cuatro cuerpos y tres niveles

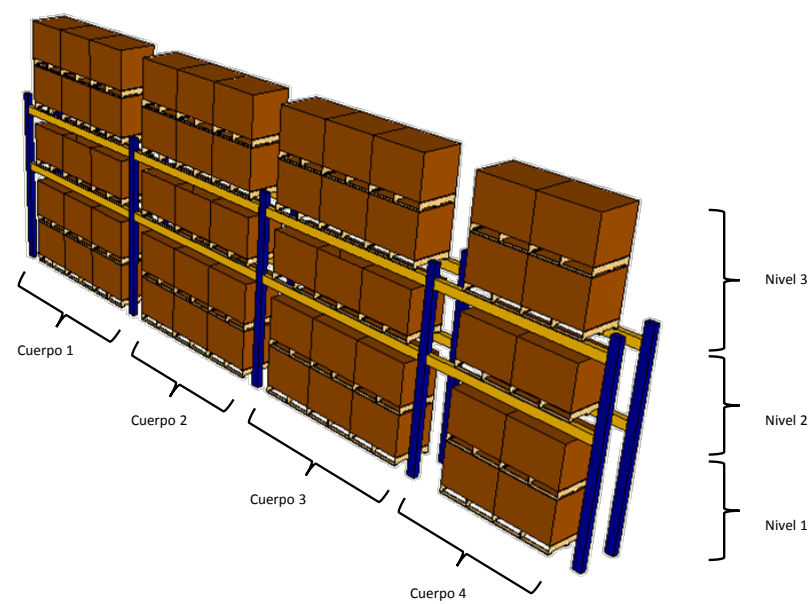

Fuente: los autores.

Número de pallets por cuerpo (NPM)

$N P M=N N C * N P N$ [pallets]

Donde:

- $N N C=$ niveles por cuerpo [niveles]

- $N P N=$ pallets por nivl [pallets/nivel]

Área del Cuerpo $(A C C)$

$A C C=L C * A C\left[\mathrm{~m}^{2}\right]$

Donde:

- $L C=$ largo del cuerpo $[\mathrm{m}]$

- $A C=$ ancho del cuerpo $[\mathrm{m}]$ 
Área del Pasillo de Almacenamiento (APA)

Donde:

$$
A P A=L P A * A P A L\left[\mathrm{~m}^{2}\right]
$$

- $L P A=$ largo del pasillo de almacenamiento $[\mathrm{m}]$

- $A P A L=$ ancho del pasillo de almacenamiento $[\mathrm{m}]$

Área Almacenamiento $(A L D)$

$A L D=\left\{\begin{array}{c}{\left[\text { Entero }\left(\frac{N E A}{N P M}\right)+1\right] * A C C+(A P A * N P)\left[m^{2}\right], N P M \neq 1 \wedge \frac{N E A}{N P M} \notin \mathbf{Z}} \\ \left(\frac{N E A}{N P M}\right) * A C C+(A P A * N P)\left[m^{2}\right], \frac{N E A}{N P M} \in \mathbf{Z}\end{array}\right.$

Donde:

- NEA = número de pallets a almacenar [pallets]

- $N P M=$ número de pallets por cuerpo [pallets]

- $N P M=$ número de pasillos

Área de Alistamiento

Área de Alistamiento (ALT)

$A L T=(N P A * A P T)+[(N P A * P A A) * A P T]+A C P\left[m^{2}\right]$

Donde:

- $N P A=$ número de pallets alistados [pallets]

- $P A A=$ porcentaje de acumulación enalistamiento [\%]

Área de Despacho

Área de Despacho $(A D)$

$A D=(N P D * A P T)+[(N P D * P A D) * A P T]+A C P\left[m^{2}\right]$

Donde:

- $N P D=$ número de pallets a despachar [pallets]

- $P A D=$ porcentaje de acumulación en despacho [\%]

Área del Pasillo de Oficina (APO)

$$
A P A=L P O * A P O L\left[\mathrm{~m}^{2}\right]
$$

Donde:

- $\angle P O=$ largo del pasillo de oficina $[\mathrm{m}]$

- $A P O L=$ ancho del pasillo de oficina $[\mathrm{m}]$
Área de Oficinas

Área de Oficinas (AOF)

$$
A O F=(N P T * A P T)+A P A\left[\mathrm{~m}^{2}\right]
$$

Donde:

- NPT = número de puestos de trabajo [puesto]

- $A P T=$ área del puesto de trabajo $\left[\mathrm{m}^{2} /\right.$ puesto]

\section{Cálculo de personal}

El cálculo de personal se fundamenta en lo mostrado en la figura 9.

Figura 9.Cálculos realizados con respecto al personal

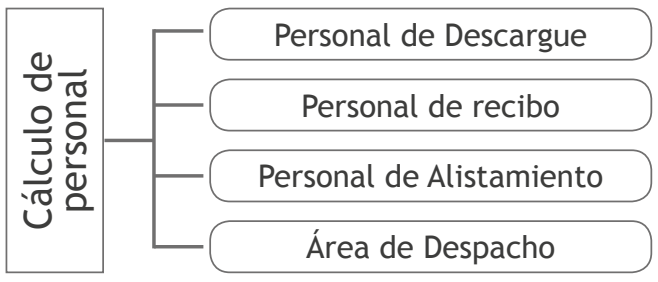

Fuente: elaboración propia. 
Personal de Descargue

Número de cajas a descargar por turno (NDP)

$N D P=(N V * N C V)[$ cajas $/$ turno $]$

Donde:

- $N V=$ número de vehículos por turno de trabajo [vehículos/turno]

- $N C V$ = número de cajas por vehículo [cajas/ vehículo]

Personal de Descargue (PD)

$P D=\left\{\begin{array}{c}{\left[\text { Entero }\left(\frac{N D P}{T P}\right)+1\right]\left[\text { operario], } T P \neq 1 \wedge \frac{N D P}{T P} \notin \mathbf{Z}\right.} \\ \left(\frac{N D P}{T P}\right) \text { [operario], } \frac{N D P}{T P} \in \mathbf{Z}\end{array}\right.$

Donde:

- $T P=$ número de cajas descargadas por un operario en turno [cajas/(turno*operario)]

Personal de Recibo

Personal de Recibo (PR)

$P R=\left\{\begin{array}{c}{\left[\text { Entero }\left(\frac{N D P}{T P R}\right)+1\right]\left[\text { [operario], } T P R \neq 1 \wedge \frac{N D P}{T P R} \notin \mathrm{Z}\right.} \\ \left(\frac{N D P}{T P R}\right) \text { [operario], } \frac{N D P}{T P R} \in \mathrm{Z}\end{array}\right.$

Donde:

- $T P R=$ número de cajas recibidas por un operario en turno [cajas/(turno*operario)]
Número de cajas a procesar por turno (NCP)

$N C P=(N O * N C O)[$ cajas $/$ turno $]$

Donde:

- $N O$ = número de órdenes por turno de trabajo [órdenes/turno]

- $N C O$ = número de cajas por orden [cajas/órdenes]

Personal de Alistamiento

Personal de Alistamiento(PA)

$P A=\left\{\begin{array}{c}{\left[\text { Entero }\left(\frac{N C P}{T P S}\right)+1\right]\left[\text { operario], } T P S \neq 1 \wedge \frac{N C P}{T P S} \notin \mathbf{Z}\right.} \\ \left(\frac{N C P}{T P S}\right) \text { [operario], } \frac{N C P}{T P S} \in \mathbf{Z}\end{array}\right.$

Donde:

- TPS = número de cajas servidas por un operario en turno [cajas/(turno*operario)]

Personal de Despacho

Personal de Despacho (PDD)

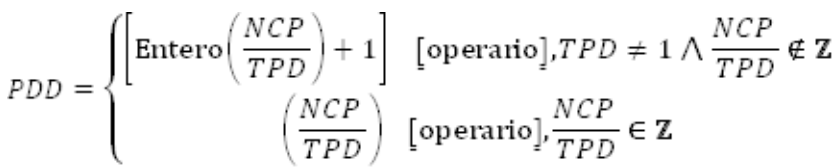

Donde:

- $T P D=$ número de cajas despachadas por un operario en turno [cajas/(turno*operario)] 


\section{RESULTADOS}

Los cálculos de capacidades considerados en el numeral anterior, se aplicaron en el caso de un operador

logístico representativo en el mercado colombiano, como se muestra a manera de esquema en la figura 10.

Figura 10. Aplicación del caso de estudio

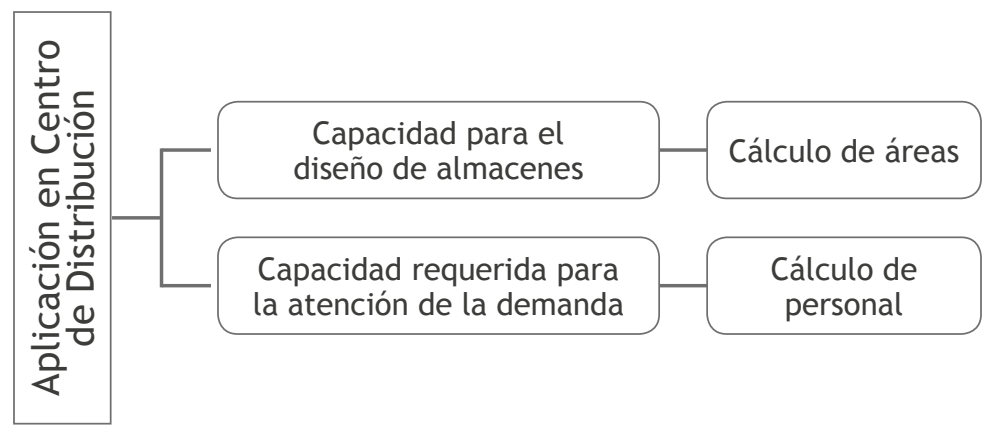

Fuente: elaboración propia.

Aplicación en áreas de centro de distribución

A continuación se muestran los cálculos realizados para un centro de distribución del operador logístico con respecto al cálculo de áreas:

Área de recibo

Área del Pallet $(A P T)$

$$
A P T=1,20 * 1=1,20\left\lfloor\mathrm{~m}^{2} / \text { pallets }\right\rfloor
$$

Donde:

- $L E=1,20[\mathrm{~m}]$

- $A E=1,00[\mathrm{~m}]$

Área de Circulación del Pasillo $(A C P)$

$$
A C P=18 * 2=36\left[\mathrm{~m}^{2}\right]
$$

Donde:

- $L P=18[\mathrm{~m}]$

- $A P=2[\mathrm{~m}]$

Área de Recibo $(A R)$ $A R=(300 * 1,20)+[(300 * 20 \%) * 1,20]+36=468\left[\mathrm{~m}^{2}\right]$
Donde:

- $N P R=300$ [pallets]

- $\mathrm{PAR}=20$ [\%]

Área de auditoría

Área de Auditoría $(A A)$

$A A=(150 * 1,20)+[(150 * 10 \%) * 1,20]+36=234\left[\mathrm{~m}^{2}\right]$

Donde:

- NPA $=150$ [pallets]

- $\mathrm{PAA}=10[\%]$

Área de almacenamiento

Número de pallets por cuerpo (NPM)

$$
N P M=7 * 2=14 \text { [pallets] }
$$

Donde:

- $N N C=7$ [nivel]

- NPN $=2$ [pallets/nivel]

Área del Cuerpo $(A C C)$

$$
A C C=2,44 * 1,05=2,562\left[\mathrm{~m}^{2}\right]
$$


Donde:

- $L C=2,44[\mathrm{~m}]$

- $\mathrm{AC}=1,05[\mathrm{~m}]$

Área del Pasillo de Almacenamiento (APA)

$$
A P A=40,04 * 3=120,12\left[\mathrm{~m}^{2}\right]
$$

Donde:

- $L P A=40,04[\mathrm{~m}]$

- $\mathrm{APAL}=3[\mathrm{~m}]$

Área Almacenamiento (ALD)

$$
A L D=(22 * 2,562)+(120,12 * 1)=176,48\left[\mathrm{~m}^{2}\right]
$$

Donde:

- $N E A=300$ [pallets]

- $N P M=14$ [pallets]

- $\mathrm{NP}=1$

Área de alistamiento

Área de Alistamiento (ALT)

$A L T=(150 * 1,20)+[(150 * 5 \%) * 1,20]+36=225\left[\mathrm{~m}^{2}\right]$

Donde:

- $N P A=150$ [pallets]

- $P A A=5[\%]$

Área de despacho

Área de Despacho $(A D)$

$A D=(142 * 1,20)+[(142 * 15 \%) * 1,20]+36=231,96\left[\mathrm{~m}^{2}\right]$

Donde:

- NPD = 142 [pallets]

- $P A D=15[\%]$

Área de oficinas

Área del Pasillo de Oficina (APO)

Donde:

$$
A P A=10 * 3=30\left[\mathrm{~m}^{2}\right]
$$

- $L P O=10[\mathrm{~m}]$

- $A P O L=3[\mathrm{~m}]$
Área de Oficinas (AOF)

$$
A O F=(3 * 1,80)+30=35,4\left[\mathrm{~m}^{2}\right]
$$

Donde:

- NPT = número de puestos de trabajo [puesto]

- $A P T=$ área del puesto de trabajo $\left[\mathrm{m}^{2} /\right.$ puesto $]$

Aplicación para personal en centro de distribución

A continuación se muestran los cálculos realizados para un centro de distribución del operador logístico con respecto al personal requerido:

Personal de descargue

Número de cajas a descargar por turno (NDP)

$$
N D P=(15 * 300)=45000[\text { cajas } / \text { turno }]
$$

Donde:

- $N V=15$ [vehículos/turno]

- $N C V=300$ [cajas/vehículo]

Personal de Descargue (PD)

$$
P D=\frac{45000}{4000}=11,25 \approx 12 \text { [operario] }
$$

Donde:

- $T P=4000$ [cajas/(turno*operario)]

Personal de recibo

Personal Recibo $(P R)$

Donde:

$$
P R=\frac{45000}{2400}=18,75 \approx 19 \text { [operario] }
$$

- $T P R=2400$ [cajas $/($ turno*operario) $]$

Número de cajas a procesar por turno $(N C P)$

$$
N C P=(54 * 100)=5400[\text { cajas } / \text { turno }]
$$

Donde:

- $N O=54$ [órdenes/turno]

- $N C O=100$ [cajas/órdenes] 
Personal de alistamiento

Personal de Alistamiento $(P A)$

$$
P A=\frac{5400}{800}=6,75 \approx 7 \text { [operario] }
$$

Donde:

- TPS $=800$ [cajas/(turno*operario)]

\section{Personal de despacho}

Personal de Despacho (PDD)

Donde:

$$
P D D=\frac{5400}{1500}=3,6 \approx 4 \text { [operario] }
$$

- $T P D=1500$ [cajas $/($ turno*operario $)$ ]

Los principales resultados consolidados para el cálculo de áreas se muestran en la tabla 2.

Tabla 2. Resultado de áreas

\begin{tabular}{|c|l|c|}
\hline VARIABLE & \multicolumn{1}{|c|}{ DESCRIPCIÓN } & RESULTADO $\left(\mathrm{M}^{2}\right)$ \\
\hline AR & Área Recibo & 468 \\
\hline AA & Área de Auditoria & 234 \\
\hline ALD & Área de Almacenamiento & 176,48 \\
\hline ALT & Área de Alistamiento & 225 \\
\hline AD & Área de Despacho & 231,96 \\
\hline AOF & Área de Oficinas & 35,4 \\
\hline & Total área & 927 \\
\hline
\end{tabular}

Fuente: elaboración propia.

Los principales resultados consolidados para el cálculo de personal se muestran en la tabla 3.

Tabla 3. Resultado de personal

\begin{tabular}{|c|l|c|}
\hline VARIABLE & \multicolumn{1}{|c|}{ DESCRIPCIÓN } & $\begin{array}{c}\text { RESULTADO } \\
\text { (OPERARIOS) }\end{array}$ \\
\hline PD & Personal de Descargue & 12 \\
\hline PR & Personal Recibo & 19 \\
\hline PA & Personal de Alistamiento & 7 \\
\hline PDD & Personal de Despacho & 4 \\
\hline & Total personal & 42 \\
\hline
\end{tabular}

Fuente: elaboración propia.

\section{CONCLUSIONES Y RECOMENDACIONES}

Mediante el cálculo de áreas y del personal requerido en el desarrollo de las operaciones logísticas en Centro de Distribución, los prestadores de estos servicios pueden planear con mayor efectividad los recursos a asignar de acuerdo con la demanda a atender desde el recibo hasta el despacho de los productos, así mismo, obtener un mejor resultado en términos de costos logísticos.

El modelo planteado favorece los procesos de planeación en los niveles táctico y operativo del prestador de servicios logísticos, principalmente en cuanto al cálculo de capacidades, infraestructura y personal operativo, lo que tiene un impacto directo en el cumplimiento de la promesa de servicio a los clientes.

Por medio de la aplicación en el operador logístico, se encuentran oportunidades de mejora en el análisis de las capacidades del personal y el diseño de instalaciones, ya sea por medio de optimización de los flujos de materiales, capacitaciones y entrenamientos, planes de formación en tareas específicas y análisis de herramientas y técnicas empleadas en los procesos logísticos; lo anterior sin dejar a un lado las metas de cumplimiento al cliente con respecto a la calidad de las entregas de los productos.

\section{REFERENCIAS}

1. Chase, R. B., Jacobs, R. F., y Aquilano, N. J. (2009). Administración de la producción y operaciones para una ventaja competitiva (12a ed.). México D.F.: McGraw-Hill.

2. Chopra, S., y Meindl, P. (2008). Administración de la cadena de suministro. Estrategia, planeación y operación (3a ed.). México D.F.: Pearson Education.

3. Collier, D. A., y Evans, J. R. (2009). Administración de operaciones. Bienes, servicios y cadena de valor ( $2 a$ ed.). México D.F.: Cengage Learning.

4. Heizer, J., y Render, B. (2009). Dirección de la producción y de operaciones. Decisiones estratégicas (7a ed.). México D.F.: Pearson Education. 
5. Hopp, W. J., y Spearman, M. L. (2008). Factory physics (3a ed.). New York: McGraw-Hill.

6. Kalenatic, D., y Blanco, L. E. (1993). Aplicaciones computacionales en producción. Bogotá: Fondo editorial UDFJC.

7. Krajewski, L. J., Ritzman, L. P., y Malhotra, M. K. (2008). Administración de operaciones, Procesos y cadena de valor (8a ed.). México D.F.: Pearson education.
8. Mora García, L. A. (2011). Gestión logística en centros de distribución, bodegas y almacenes (1a ed.). Bogotá: Ecoe Ediciones.

9. Sipper, D., y Bulfin, R. L. (1997). Planeación y control de la producción (1a ed.). México D.F.: McGraw-Hill.

10. Suppla S.A. (2014). Diccionario Suppla de logística y transporte (1a ed.). Bogotá: LID Editorial Colombia. 